\title{
Liquid Wax Esters in Liver Oils of the Deep-Sea Teleost Fish Laemonema longipes
}

\author{
Kenji Hayashi* \\ (Accepted July 31, 1987)
}

\begin{abstract}
The content and the constituent fatty alcohols and acids of wax esters in liver oils of the deepsea teleost fish Laemonema longipes from the northwestern Pacific Ocean were investigated.

An analysis of forty specimens revealed that their liver weights $(48.9 \pm 14.9 \mathrm{~g})$ accounted for $10.3 \pm 1.9 \%$ of their total body weight; the livers consisted of $74.0 \pm 6.3 \%$ oils. The liver oils contained high quantities of wax esters $(73.6 \pm 11.6 \%)$ and low proportions of triglycerides $(25.1 \pm$ $11.5 \%$, and also significant amounts of unsaponifiable materials $(35.4 \pm 5.7 \%$ ), almost entirely consisting of fatty alcohols $(98.8 \pm 0.5 \%)$. The relationship between the amount of wax esters in the liver $(\mathrm{Y}, \mathrm{g})$ and liver weight $(\mathrm{X}, \mathrm{g})$ was expressed by the following equation: $\mathrm{Y}=3.241+0.477 \mathrm{X}$, $(r=0.812)$.

The component fatty alcohols of wax esters were found to consist mostly of monoenes ( $86.5 \pm$ $4.2 \%$ ) and saturates $(13.4 \pm 4.1 \%)$ of 14 to 24 carbon numbers. The principal components found were: $22: 1(47.4 \pm 4.8 \%), 20: 1(27.3 \pm 2.6 \%)$ and $16: 0(9.5 \pm 2.8 \%)$ alcohols. On the other hand, the wax ester fatty acids contained $18: 1(26.9 \pm 3.6 \%), 16: 1(15.3 \pm 1.1 \%), 20: 5 \omega 3(9.4 \pm$ $2.7 \%)$, and $16: 0(9.3 \pm 0.8 \%)$ acids, as their major components.

It was concluded that the potential exploitation of $L$. longipes liver oil, unusually rich in liquid wax esters, appears as a possible substitute of sperm whale oil.
\end{abstract}

Wax esters have been used as the characteristic basis for a wide range of products from cosmetics to extreme-pressure and temperature lubricants, and could also be valuable as a source of fatty alcohols for the chemical industry. As the shortage of sperm whale oil, rich in wax esters, is evident in near future, new sources of commercially desirable wax esters are being sought. Recently more attention has been called for the seed oil of the desert shrub, jojoba Simmondsia chinensis, ${ }^{12}$ and for the fish oil taken from a certain deep-sea species, orange roughy Hoplostethus atlanticus, ${ }^{2,3)}$ both of which contain very high concentrations of wax esters.

On the other hand, Komori and Agawa ${ }^{4}$ first discovered that the oil of a deep-sea fish Laemonema morosum contained large amounts of unsaponifiable materials, consisting mainly of 22:1 alcohol. In a previous report, we demonstrated that the liver of a deep-sea teleost fish Podonema longipes contained remarkably high amounts of oils, characterized by having a high percentage of wax esters, comprised primarily of $22: 1$ and $20: 1$ alcohols. ${ }^{5)}$ These two fishes are considered to be the same species belong to the family Moridae Laemonema longipes, which is potentially available in large quantities. Their livers are thus presently an untouched resource as another replacement of sperm whale oil. Recently, Kayama and Iijima ${ }^{6)}$ examined $L$. longipes liver oil and tried to separate wax esters by the centrifugation.

The present study attempts to describe in more detail the content of such wax esters and the composition of fatty alcohol moiety in the liver oils of a number of $L$. longipes differing in growth size, caught in the northwestern Pacific Ocean. The component fatty acids of the wax esters were also determined.

\section{Experimental}

\section{Materials}

Forty $L$. longipes specimens, ranging from 292.0 to $710.0 \mathrm{~g}$ (mean \pm standard deviation: $469.2 \pm 93.8 \mathrm{~g}$ ) in body weight, were caught at a depth of ca. $700 \mathrm{~m}$ in the northwestern Pacific Ocean in August, 1985. They were stored at $-20^{\circ} \mathrm{C}$ until analysis. The livers of this species were used individually for lipid extraction.

\section{Lipid Extraction and Analysis}

The liver oils were extracted from the tissues by

* Faculty of Fisheries, Hokkaido University, Hakodate, Hokkaido 041, Japan (林 賢治: 北海道大学水産 学部). 
the method of Bligh and Dyer. ${ }^{7)}$ Wax esters were then fractionated from the liver oils by thinlayer chromatography. The liver oils and wax esters were subjected to hydrolysis in ethanolic potassium hydroxide by boiling under reflux for $1 \mathrm{~h}$. The unsaponifiable material containing fatty alcohols was isolated from the fatty acids by repetitive diethyl ether extraction from the aqueous alkaline solutions. The fatty acids were recovered from the each saponification mixture by a routine method. The quantitative analyses of liver oils and unsaponifiable materials were performed by employing the thin-layer chromatography - flame ionization detector method using an Iatroscan TH-10 instrument with Chromarod S-II rods. Chromatograms were recorded and integrated by a Shimadzu Chromatopack R-1A. Chromarod S-II rods, spotted with a chloroform solution containing liver oils or unsaponifiable materials, were developed in hexane-diethyl ether -formic acid (92:8:0.5 v/v/v for the former; 50:50:0.5 v/v/v for the latter). The operating conditions were as follows: flow rates of hydrogen and air were $160 \mathrm{~m} / / \mathrm{min}$ and $2000 \mathrm{~m} / / \mathrm{min}$ respectively; scan speed was $30 \mathrm{~s} / \mathrm{scan}$.

\section{Thin-Layer Chromatography (TLC)}

A preliminary qualitative analysis of the liver oil or its unsaponifiable material was performed using TLC. The fractionation of liver oil wax esters was carried out on prepared plates. A portion of the fatty alcohol acetates was fractionated, according to their degree of unsaturation, on silicic acid plates impregnated with $15 \%$ silver nitrate. Thin-layers of silicic acid, $0.25 \mathrm{~mm}$ thick $(20 \times 20 \mathrm{~cm})$, were used for analytical and preparative purposes. Hexane-diethyl ether-acetic acid (90:10:1 v/v/v for liver oils and 40:60:1 $v / v / v$ for unsaponifiable materials) were used as developing solvents. After development, the plates were sprayed with $50 \%$ sulfuric acid or alcoholic rhodamine-6-GO as visual reagents. Lipid constituents were identified by comparison of $R f$ values with those of corresponding standards.

\section{Derivatization}

Fatty alcohols were converted into acetates by dissolving them in a mixture of pyridine and acetic anhydride, and were left to stand in darkness at room temperature overnight. Fatty acids were methylated with $14 \%$ boron trifluoride in methanol. Prior to the gas liquid chromatography, further purification of these derivatives was carried out by TLC.

\section{Hydrogenation}

Samples were dissolved in hexane containing $5 \%$ palladium catalyst, and treated with hydrogen at room temperature and under atomospheric pressure for $1 \mathrm{~h}$ to achieve complete hydrogenation.

\section{Gas Liquid Chromatography (GLC)}

Analysis by GLC was carried out using a Shimadzu model GC 8APF gas chromatograph equipped with a dual hydrogen flame ionization detector. The acetates of fatty alcohols and the methyl esters of fatty acids were analyzed on $1.5 \mathrm{~m}$ $\times 3 \mathrm{~mm}$ id. glass columns packed with Unisol 3000 on Uniport C (80/100 mesh) and with $10 \%$ DEGS on Chromosorb W AW (80/100 mesh), respectively. The operating conditions were as follows: column temperatures were $225^{\circ} \mathrm{C}$ for the acetates of fatty alcohols, and $180^{\circ} \mathrm{C}$ for the methyl esters of fatty acids. Nitrogen was used as a carrier gas. The components' identity for fatty alcohols and acids was confirmed by comparison with available known standards as well as by making semilogarithmic plots of the retention times against the carbon numbers with different degrees of double bonds. A quantitative analysis was performed on the basis of the percentage of the total area using a Shimadzu model R-3A Chromatopack.

\section{Results and Discussion}

\section{Content of Liver Oil}

An examination of the forty $L$. longipes specimens revealed their liver weights ranging from 16.5 to $90.0 \mathrm{~g}(48.9 \pm 14.9 \mathrm{~g}$ as mean); the relative weight of the liver accounted for 5.7 to $13.7 \%(10.3 \pm 1.9 \%)$ of the total body weight. The relationship between liver weight $(Y, g)$ and total body weight $(\mathrm{X}, \mathrm{g})$ was expressed by the following equation: $Y=-12.408+0.131 X,(\mathrm{I}=$ 0.820 ).

The livers yielded 11.1 to $65.5 \mathrm{~g}(36.4 \pm 12.1 \mathrm{~g})$ of oils; the relative weight of the liver oils amounted to 50.1 to $82.9 \%(74.0 \pm 6.3 \%)$ of the liver weight and 3.8 to $10.6 \%(7.7 \pm 1.6 \%)$ of the total body weight, respectively. As seen in Fig. 1, the relationship between the amount of liver oils $(Y$, g) and liver weight $(X, g)$ could be expressed as follows: $Y=-1.350+0.772 X,(r=0.956)$.

\section{Component of Liver Oil and Unsaponifiable Material} TLC of the $L$. longipes liver oil revealed relatively 


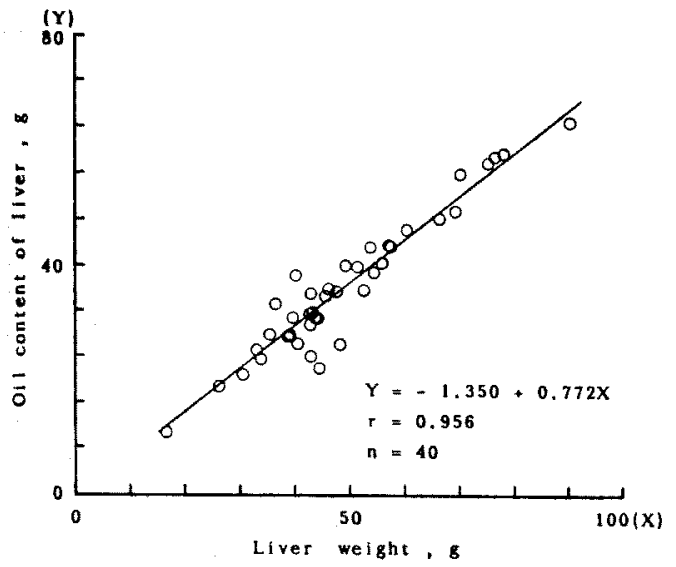

Fig. 1. Relationship between the amount of liver oils $(\mathrm{Y}, \mathrm{g})$ and liver weight $(\mathrm{X}, \mathrm{g})$ of the examined L. longipes.

Table 1. Lipid class composition of liver of the examined $L$. longipes

\begin{tabular}{lc}
\hline \hline Component & Mean \pm SD $\%^{*}$ \\
\hline Wax esters & $73.6 \pm 11.6$ \\
Triglycerides & $25.1 \pm 11.5$ \\
Fatty acids & $0.3 \pm 0.1$ \\
Sterols & $0.2 \pm 0.1$ \\
Partial glycerides & $0.2 \pm 0.1$ \\
Phospholipids & $0.7 \pm 1.1$ \\
\hline
\end{tabular}

* $N=40$.

large spots corresponding to wax esters and triglycerides. The composition of the liver oils is presented in Table 1. The liver oils were characterized by a high level of $73.6 \pm 11.6 \%$ wax esters, followed by $25.1 \pm 11.5 \%$ triglycerides. It is noteworthy that even the small-sized $L$. longipes examined had an abundant amount of wax esters in their livers. The wax ester content in the liver oils of the present $L$. longipes was comparatively higher than that reported previously $(47.7 \%){ }^{3}$ )

The wax ester content of the livers examined in this study ranged from 7.6 to $47.9 \mathrm{~g}(26.5 \pm 8.8 \mathrm{~g})$. The relationship between the amount of wax esters in the liver $(\mathrm{Y}, \mathrm{g})$ and liver weight $(\mathrm{X}, \mathrm{g})$ was expressed by the following equation: $\mathrm{Y}=$ $3.241+0.477 \mathrm{X},(\mathrm{r}=0.812)$ (Fig. 2). Thus, substituting the weight of $L$. longipes liver for this expression, it is possible to estimate the absolute amount of wax ester of the individual liver.

The liver oils were also characterized by a high high level of unsaponifiable materials ranging from 23.2 to $48.4 \%(35.4 \pm 5.7 \%)$. The content of unsaponifiable material in the livers accounted

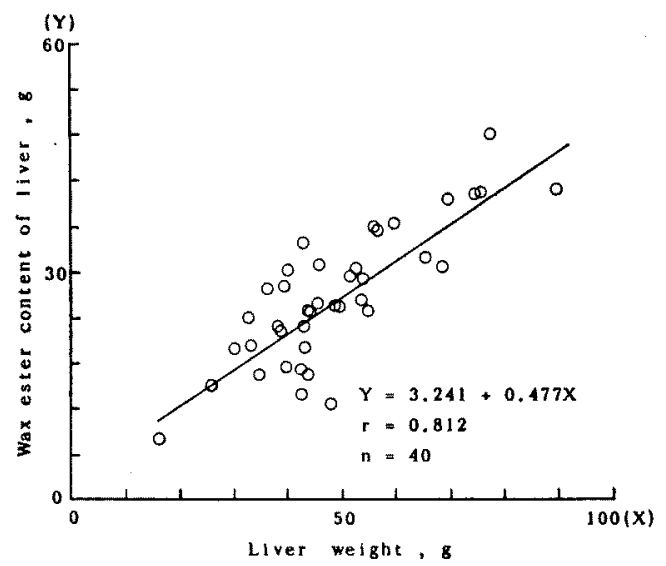

Fig. 2. Relationship between the amount of wax esters in the liver $(Y, g)$ and liver weight $(X, g)$ of the examined L. longipes.

Table 2. Composition of unsaponifiable materials of liver oils of the examined $L$. longipes

\begin{tabular}{lc}
\hline \multicolumn{1}{c}{ Component } & Mean \pm SD \%* \\
\hline Fatty alcohols & $98.8 \pm 0.5$ \\
Sterols & $0.7 \pm 0.3$ \\
Glyceryl ethers & $0.2 \pm 0.3$ \\
Methoxy glyceryl ethers & $0.1 \pm 0.1$ \\
Polar compounds & $0.2 \pm 0.1$ \\
\hline$* \mathrm{~N}=40$. &
\end{tabular}

for 3.6 to $22.9 \mathrm{~g}(12.8 \pm 4.2 \mathrm{~g})$. The constituents of unsaponifiable materials are as shown in Table 2. The unsaponifiable materials were almost entirely fatty alcohols $(98.8 \pm 0.5 \%)$. Small quantities of sterols and glyceryl ethers were also detected. The content of the fatty alcohols in the livers ranged from 3.6 to $22.6 \mathrm{~g}(12.6 \pm 4.2 \mathrm{~g})$. The relationship between the amount of fatty alcohols in the liver $(\mathrm{Y}, \mathrm{g})$ and liver weight $(\mathrm{X}, \mathrm{g})$ could be derived as follows: $Y=1.549+0.227 \mathrm{X}$, $(r=0.808)$. TLC revealed that the small spot migrating slower than the glyceryl ether spot on the silicic acid plate corresponded to that of methoxy glyceryl ethers isolated from ratfish liver lipids. ${ }^{8)}$

\section{Composition of Fatty Alcohol and Acid of Wax Ester}

The component fatty alcohols of the liver oil wax esters are as shown in Table 3. Even numbers of carbon atoms, ranging from $\mathrm{C}_{14}$ to $\mathrm{C}_{24}$, were abundant as compared with odd ones. The most predominant component found was $22: 1$ alcohol $(47.4 \pm 4.8 \%)$, followed by $20: 1(27.3 \pm 2.6 \%)$ and 
16: $0(9.5 \pm 2.8 \%)$ alcohols, indicating a very high concentration of monoenoic $(86.5 \pm 4.2 \%)$. Branched fatty alcohols also occurred but only in

Table 3. Fatty alcohol composition of wax esters in liver oils of the examined $L$. longipes

\begin{tabular}{cc}
\hline Component & Mean \pm SD \%*2 \\
\hline $14: 0$ & $2.3 \pm 1.1$ \\
$15: 0$ & $0.2 \pm 0.1$ \\
$16: 0$ & $9.5 \pm 2.8$ \\
$17: 0$ & $0.2 \pm 0.1$ \\
$18: 0$ & $0.7 \pm 0.1$ \\
$19: 0$ & $0.2 \pm 0.1$ \\
$20: 0$ & $0.3 \pm 0.1$ \\
$16: 1$ & $4.6 \pm 1.3$ \\
$17: 1$ & $0.1 \pm 0.1$ \\
$18: 1$ & $2.6 \pm 0.6$ \\
$19: 1$ & $0.1 \pm 0.1$ \\
$20: 1$ & $27.3 \pm 2.6$ \\
$21: 1$ & $0.1 \pm 0.1$ \\
$22: 1$ & $47.4 \pm 4.8$ \\
$23: 1$ & $0.2 \pm 0.1$ \\
$24: 1$ & $4.2 \pm 0.7$ \\
$15: 0$ br*3 & $0.1 \pm 0.1$ \\
$16: 0$ br & $0.1 \pm 0.1$ \\
\hline Saturates & $13.4 \pm 4.1$ \\
Monoenes & $86.5 \pm 4.2$ \\
Branched & $0.1 \pm 0.2$ \\
\hline No. of carbon atoms: no. of double bonds. & \\
& \\
&
\end{tabular}

Table 4. Fatty acid composition of wax esters in liver oils of the examined $L$. longipes

\begin{tabular}{cc}
\hline Component*1 & Mean + SD \%*2 \\
\hline $14: 0$ & $6.8 \pm 1.3$ \\
$16: 0$ & $9.3 \pm 0.8$ \\
$18: 0$ & $1.3 \pm 0.2$ \\
$14: 1$ & $0.5 \pm 0.2$ \\
$16: 1$ & $15.3 \pm 1.1$ \\
$17: 1$ & $1.7 \pm 0.2$ \\
$18: 1$ & $26.9 \pm 3.6$ \\
$20: 1$ & $9.6 \pm 2.6$ \\
$22: 1$ & $7.6 \pm 2.9$ \\
$18: 2 \omega 6$ & $1.8 \pm 0.3$ \\
$18: 4 \omega 3$ & $2.4 \pm 0.6$ \\
$20: 4 \omega 6$ & $0.2 \pm 0.1$ \\
$20: 4 \omega 3$ & $0.9 \pm 0.3$ \\
$20: 5 \omega 3$ & $9.4 \pm 2.7$ \\
$22: 6 \omega 3$ & $1.5 \pm 0.4$ \\
\hline Saturates & $18.3 \pm 2.0$ \\
Monoenes & $62.4 \pm 4.1$ \\
Polyenes & $18.7 \pm 3.7$ \\
Unknowns & $0.5 \pm 0.5$ \\
\hline
\end{tabular}

*1 No. of carbon atoms: no. of double bonds. *2 $\mathbf{N}=40$. small amounts. However, no polyunsaturated fatty alcohols were detected.

The component fatty alcohols were in good agreement in composition to those of the same species reported previously. ${ }^{5}$ ) It should be noted that the aforementioned fatty alcohols obtained from the liver oil wax esters of $L$. longipes were extremely different in composition to those of the jojoba seed oil, ${ }^{1)}$ orange roughy oil, ${ }^{2,3)}$ and sperm whale oil. ${ }^{32}$

The major fatty acid composition of the $L$. longipes wax esters studied is given in Table 4. As shown in Table 4, 18:1, 16:1, 20:1, 20:5 53 , and 16: 0 acids were characteristic, and their contents were $26.9 \pm 3.6 \%, 15.3 \pm 1.1 \%, 9.6 \pm 2.6 \%$, $9.4 \pm 2.7 \%$, and $9.3 \pm 0.8 \%$ of the total fatty acids, respectively. The high proportion of $20: 5 \omega 3$ acid in the L. longipes wax esters were extremely different from the wax ester fatty acids found in jojoba seed oill, ${ }^{1)}$ orange roughy oil, ${ }^{2,3)}$ and sperm whale oil, ${ }^{3)}$ which were lacking of this polyunsaturated fatty acid.

Although the extent of the deep-sea teleost fish $L$. longipes resource is at present unknown, it is large (past catches: $14,000 \mathrm{t}$ in 1985), and it is possible that a fishery could be developed to process these fish. It can be concluded therefore that the potential exploitation of $L$. longipes liver oil, unusually rich in liquid wax esters, appears as a possible substitute of sperm whale oil.

\section{Acknowledgments}

The author wish to thank Mr. M. Sano for his assistance in the cource of the present study. This work was supported in part by a grant from the Ministry of Education.

\section{References}

1) T. K. Miwa: J. Amer. Oil Chem. Soc., 61, 407410 (1982).

2) K. Hayashi and T. Takagi: Nippon Suisan Gakkaishi, 46, 459-463 (1980).

3) D. H. Buisson, D. R. Body, G. J. Dougherty, L. Eyres, and P. Vlieg: J. Amer. Oil Chem. Soc., 59, 390-395 (1982).

4) S. Komori and T. Agawa: J. Amer. Oil Chem. Soc., 32, 525-528 (1955).

5) K. Hayashi and M. Yamada: Bull. Fac. Fish. Hokkaido Univ., 26, 356-366 (1976).

6) M. Kayama and N. Iijima: in "Suisanbutsu Kakoriyo Gijutsu kenkyu Kaihatsu Kenkyu Seika no Gaiyo", Suisancho Kenkyubu Kenkyuka, 1983, pp. 142-150. 
7) E. G. Bligh and W. J. Dyer: Can. J. Biochem. Physiol., 37, 911-917 (1959).
8) K. Hayashi and T. Takagi: Nippon Suisan Gakkaishi, 48, 1345-1351 (1982).

Nippon Suisan Gakkaishi : Formerly Bull. Japan. Soc. Sci. Fish. 